\title{
PRACTICAL APPROACH TO EXPERIMENTATION IN A SIMULATION STUDY
}

\author{
Benny Tjahjono \\ Raúl Fernández \\ Manufacturing Department \\ School of Applied Sciences \\ Cranfield University \\ Cranfield, Bedford, MK43 0AL, UK
}

\begin{abstract}
Simulation study of complex production facilities can be a challenging task for manufacturing engineers as it requires skills to build the models and to conduct experiments. Accurate modeling but inadequate experimentation may lead to poor decision and can be detrimental particularly when financial investment is involved. This paper proposes a practical approach to simulation experimentation in the context of simulation study of an engine assembly line. The overall aim of the study was to increase the productivity and efficiency of the line. The approach was deployed in the form of a methodology that was used to select the most feasible outcome from a series of simulation experiments, taking into account the minimum effort/investment needed to implement the improvement.
\end{abstract}

\section{INTRODUCTION}

Intense competition in the automotive market has put pressure on manufacturers to continuously improve their manufacturing processes in order to increase the throughput while maintaining highly efficient operations so that the unit cost can be reduced. Design and redesign of manufacturing systems and processes, however, can be daunting for manufacturing engineers. In the case of an assembly line, for instance, due to the complexity, stochastic nature and interconnectedness between machines and other components within the line, it is difficult to predict the likely performance of the line without tools such as computer-based simulation.

The car manufacturer upon which this study was based has identified the need to improve the productivity of one of its engine assembly lines. The overall purpose of the study was to investigate the possible causes for this low productivity and ultimately to improve efficiency of the line. The company is aware that poor design can affect the production capacity and more importantly wrong decision can be detrimental to the whole business. As physical ex- periments with the actual line are extremely risky and costly, the company has applied simulation to many of the decision making processes.

Simulation study is intrinsically a demanding and resource intensive task involving at least two major activities: model building and experimentation. Model building requires the modelers to fully understand the problems, envisage and construct the model elements and identify the relationships that logically link those elements together (Guru and Savory 2004), but without adequate experimentation, the model will not likely to provide appropriate answers to the what-if questions commonly asked in any simulation study. As with model building, experimentation is also a challenging task.

The main aim of this paper is therefore to propose a practical approach to experimentation in the form of a methodology that can be used to help manufacturing engineers during the execution of the simulation study. To illustrate its practical applications, the methodology has been used in the context of simulation and modeling of a car engine assembly line. The goal of the simulation study is ultimately to identify potential improvements attributed to the increased productivity and improved efficiency of the line.

The paper first discusses the productivity disturbances that affect the efficiency of manufacturing systems and briefly summarizes common ways to detect them. The main body of the paper describes in detail both model building and experimentation where the methodology has been applied to improve the line. Findings, some insights and further work will conclude the paper.

\section{PRODUCTIVITY DISTURBANCES}

Disturbances affect the efficiency of manufacturing systems (Ingemansson and Bolmsjö 2004). Reducing the disturbances is therefore key to increased productivity and improved efficiency. Kuivanen (1996) defines disturbances as events that occur independently of the system's inten- 


\section{Tjahjono and Fernández}

tions. Ingemansson and Bolmsjö (2004) categorize the disturbances into downtime (planned and unplanned stops), speed and quality losses. The throughput of a production line is restricted by the capacity and performance of the elements that compose the system (Roser et al. 2001).

Disturbances are known to be the reasons for poor performance of the elements of the system (Ingemansson and Bolmsjö 2004) and one of those elements that impedes the achievement of the system is the bottleneck (Roser et al. 2001). If the bottleneck is eliminated, the performance of the whole system will improve.

Unfortunately, bottlenecks are not always straightforward to detect, although blocking and idleness of workstations, e.g. in an assembly line, are obvious signs of bottlenecks. Bottlenecks exist typically in an assembly line simply because a perfect balance between workstations in term of cycle time is not possible to achieve. Furthermore, the elimination of a bottleneck in one part of the system tends to lead to the discovery of others (Chryssolouris $2005)$ thus improvement to the line can only reduce the effect of the bottleneck, which can be done through:

- Work redistribution, e.g. layout redesign, task reduction

- Extra resources, e.g. buffers, operators, work-inprogress, or

- Changing the configuration of the bottleneck resources, e.g. cycle times.

Ingemansson et al. (2005) state that simulation is a suitable tool to analyze disturbances and bottlenecks because it is capable of facilitating the implementation of modification in a simulation model and the impacts can be demonstrated reasonably easily.

Several other researchers have also proposed practical bottleneck detection and reduction methods using simulation. Law and Kelton (1991), for example, applied the waiting time and workload of work stations concepts to detect bottlenecks, although Roser et al. (2001) doubt the effectiveness of these. They argued that it could be that the effect of buffers actually disturbs the reality. In the case of the workload of workstation, some machines may have similar workload and the difference could be difficult to spot.

For that reason, Roser et al. (2001) elaborated a method consisting of grouping the states of the entities of the model into active or inactive. Active state is when the entity is working or being repaired and inactive state is when the entity is blocked or idle. The duration of the work stations being active is measured throughout the simulation data. The workstation with the longest average active period is the bottleneck. Faget et al. (2005) applied this methodology in order to automate the bottleneck analysis which increased accuracy of the simulation results.

Roser et al. (2002) subsequently proposed another method that was able to detect the shifting momentary bottleneck and determine the bottleneck over a period of time.

\section{MODEL BUILDING}

This section discusses in detail the execution of the simulation study carried out at an engine assembly facility of a major car manufacturer in the UK. As mentioned before, the main purpose is to identify the potential disturbances that affect productivity and efficiency of the line.

\subsection{The Assembly Line}

Assembly lines are commonly used in a mass production system such as in engine production. The engine assembly line at the company consists of a number of workstations arranged in a sequence and linked by a conveyor belt. The workstations can be automated, e.g. performed by robots, or manual where the work is carried out by human operators, or even semi-automated, where an operation is carried out by a machine but maneuvered by an operator.

Car engines are mounted on specialized jigs called platens and transported along the conveyor belt to the workstations. The platens can be rotated allowing the operators to access different parts of the engines and to perform different operations. At each workstation, a task has to be completed according to a standardized processing time or also known as the cycle time. When an operator is working on that particular engine, the conveyor is usually stopped and remains stationary until the task is completed. The operator will then release the engine to the next workstation. This process is repeated along the assembly line and the whole sequence of these operations leads to the production of a complete car engine.

In the case of manual assembly operation, a workstation can take more time to complete the task causing starvation for the next workstation, or conversely, is quicker than the standardized cycle time, causing bottleneck to the next workstation. For this reason, two workstations are often decoupled by a buffer to ensure the balance of the line and smooth flow of the engines. In the case of car assembly line with conveyor belt, the capacity of the buffer is determined by the distance or length of the conveyor belt between the two workstations, which also corresponds to the amount of work-in-progress (WIP).

\subsection{The Simulation Tool}

The tool used in this simulation study is a proprietary Excel spreadsheet that acts as input/output interface to Witness Manufacturing Performance edition. The spreadsheet has been significantly enhanced with many features, one of which being the automatic generation of simulation codes that can be run by Witness.

The use of such an interface can significantly speed up model building, making it simpler for users who are not the expert in simulation to build and modify complex assembly lines models consisting of many machines, robots and op- 
erators combined for the manufacture of many engine variants. The automatic code generation has been made possible by programming macros inside Excel that link to a library of custom designed Witness modules. The library of modules consists of the equipment typically present on an engine assembly line, e.g. manual/automatic workstations, conveyors, turntables etc. Figure 1 shows the screenshot of the simulation tool where the cycle time has been transferred to corresponding Witness model.

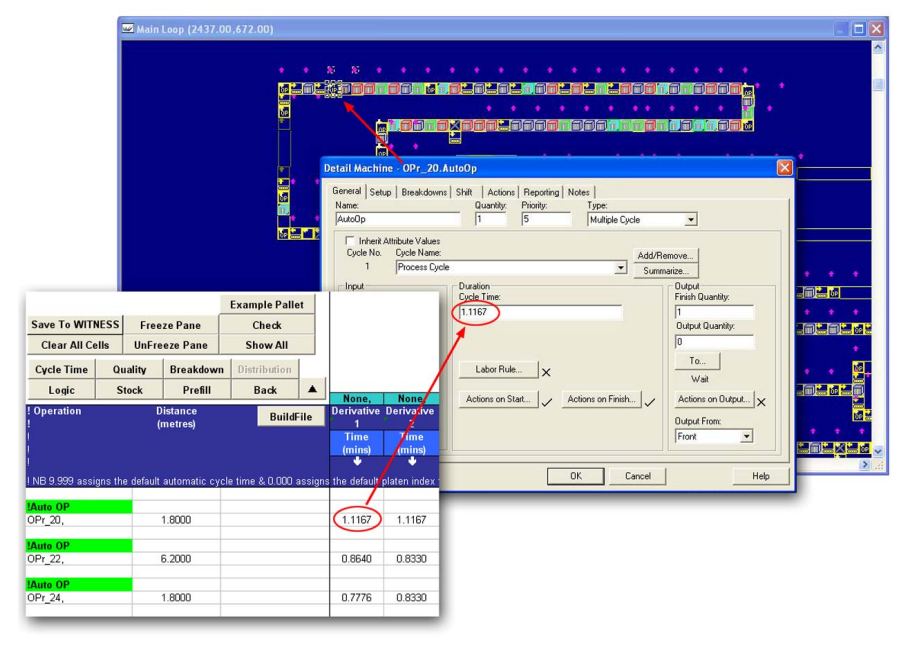

Figure 1: The simulation tool

Unlike the conceptual modeling using typical visual interactive simulation tools, building a model using this tool only requires the users to enter the input data, such as cycle times of the workstations, the output flow logic of the line, and quality and reliability data. The complete simulation model can be generated automatically by entering the data into the relevant spreadsheets and pressing the 'RUN' button. As the interface adopts the reusable component modeling principles (Winnell \& Ladbrook 2003), the main benefit is that the model can be generated much more quickly compared to developing that model from scratch using visual interactive modeling simulation tools.

\subsection{Model Verification}

Before carrying out the experiments, it is important to make sure that the model is logically correct and accurately represents the layout of the assembly line. This process is known as verification (Sargent 2004). During verification of the model, the simulation interface checks the detail of the assembly line to be modeled and tests whether the engine follows the logic and the model layout specified before generating the codes in Witness. If there is an error in the model, e.g. missing parameters, resulting in the interface not able to interpret this, then the model construction will stop and the interface gives an error message. Verification of the model also requires the calculation of effi- ciency which, according to Reid and Sanders (2005), is a metric that measures actual output relative to some standard of output, and can be denoted as:

$$
\text { Efficiency }=\frac{T}{T_{\max }} \times 100 \%
$$

where

$$
\begin{aligned}
& T= \text { throughput rate obtained from the simulation } \\
& \text { model (engine/hr) } \\
& T_{\max }=\text { theoretical maximum throughput rate (en- } \\
& \text { gine/hr) }
\end{aligned}
$$

The theoretical maximum throughput time is denoted as:

$$
T_{\max }=\frac{60}{t}
$$

where

$t=$ theoretical takt time of the balanced line (mins)

The throughput rate from the simulation model can be obtained by running so called the 'cyclic model'. This is the basic model where the parameters inserted into the workstations are cycle times only without any disturbances, e.g. machine breakdowns, quality data etc. When the breakdown and other disturbances are added into the cyclic model, the efficiency of the line will drop, as the throughput rate reduced. Maximizing the efficiency is obviously the ultimate goal of this simulation study.

\subsection{Model Validation}

Once the logic of the model is verified, the next stage is to make the simulation model more accurately represents the behavior of the actual assembly line. This is done by incorporating the disturbances which include reliability of the machines, operator performance data and quality data.

- Breakdown data. Every piece of equipment on the line occasionally breaks down. A breakdown can be anything from a simple stoppage to a major fault that needs extensive repairs from the manufacturer of the machine. Machine breakdown affects the flow of the line and therefore needs to be adequately modeled. Breakdown data can be gathered using an online data collection and monitoring system from which a distribution of breakdowns, in terms of Mean Time Between Failures (MTBF) and the associated Mean Time To Repair (MTTR), can be constructed. Figure 2 shows an example of a breakdown distribution at a workstation.

- Human behavior data. The operator's performance data are referred to as variations in operator's performance from one cycle to another. This can be the consequence of the deviation from the work standard, poor operator training, and occa- 
sionally, lack of discipline. However, poor quality of the incoming engine from previous workstation can also prevent operators from finishing their job in time. These data are collated into a distribution which approximately represents the human breakdown pattern. Work in modeling human behavior was carried out by Baines et al (2005).

- Quality data are added to the cyclic model in the form of the percentage of failure of each operation after a certain test and the reprocessing time, i.e. the time an operator spends to rectify the defected engine.

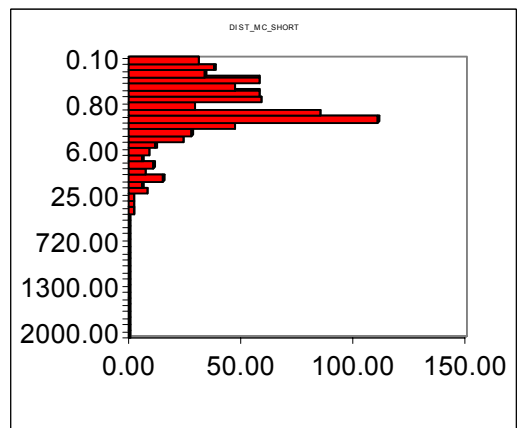

Figure 2: An example of breakdown distribution of a workstation

In this study, the model was validated by comparing its throughput after the disturbances were incorporated into the cyclic model $\left(T_{0}\right)$ against the actual throughput of the line. As a rule of thumb, $\pm 5 \%$ discrepancy was acceptable by manufacturing engineers and the model was considered valid. During the experimentation phase, $T_{0}$ was used as the reference in order to check whether or not the efficiency actually improved.

\subsection{Initial Setup}

Identifying the steady state was the first step before running the experiments. The steady state represents the stable condition of the system and therefore is used to decide the run time of the simulation model. The overall purpose of this stage was to scrutinize the long-run behavior of the model. If the run time chosen is lower than the steady state, then the outputs obtained may not be accurate. On the other hand, if the run time chosen is too long from the steady state, often the output will not be more accurate.

To identify the steady state, the model was run initially for 120 days. The output is the average of the daily throughputs. For each day, the daily throughput $\left(X_{i}\right)$ was recorded and then the average from the first day to that day was obtained by using the following formula (Nakayama 2006):

$$
\overline{X_{n}}=\frac{\sum_{i=1}^{n} X_{i}}{n}
$$

where

$n=$ number of run

Figure 3 shows that $X_{i}$ stabilizes around the $16^{\text {th }}$ day, varying only decimals after this day. This means after day 16 , the output hardly differs and it should be accurate enough to calculate the throughput of the line as long as other conditions do not change.

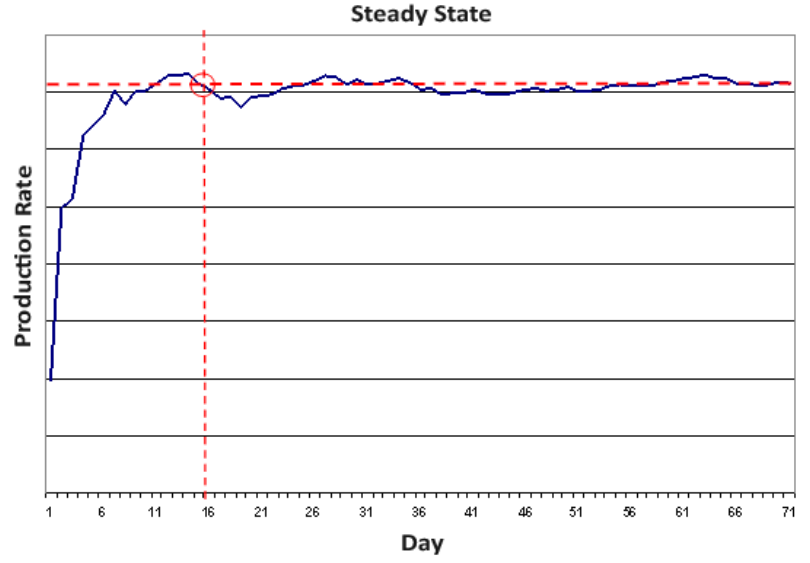

Figure 3: Steady state analysis

In addition, Figure 4 shows the difference between the final throughput $\left(\overline{X_{n}}\right)$ and $X_{i}$ for each day. It can be seen that after day 16 the variations become insignificant and the model reaches steady state after day 16 .

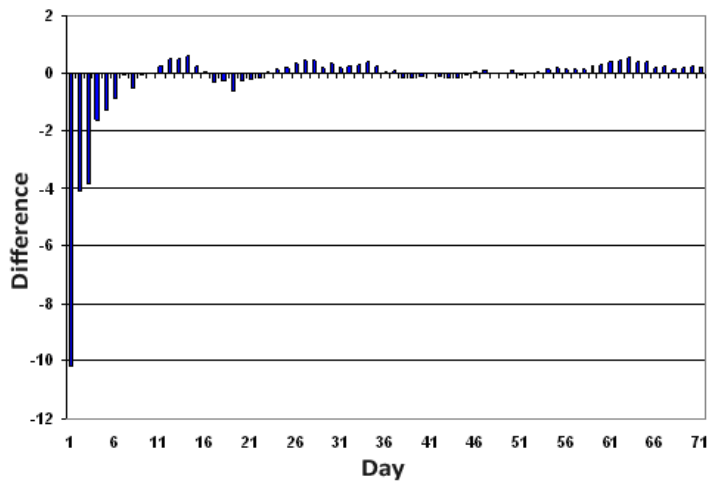

Figure 4: Differences between $\overline{X_{n}}$ and $X_{i}$

\section{EXPERIMENTATION}

As mentioned earlier, the overall goal of the simulation study was to improve efficiency of the engine assembly line. Figure 5 shows the methodology used for conducting experimentation with the model. The methodology consists of bottleneck detection, bottleneck reduction/elimination and finally efficiency improvement. 


\section{Tjahjono and Fernández}

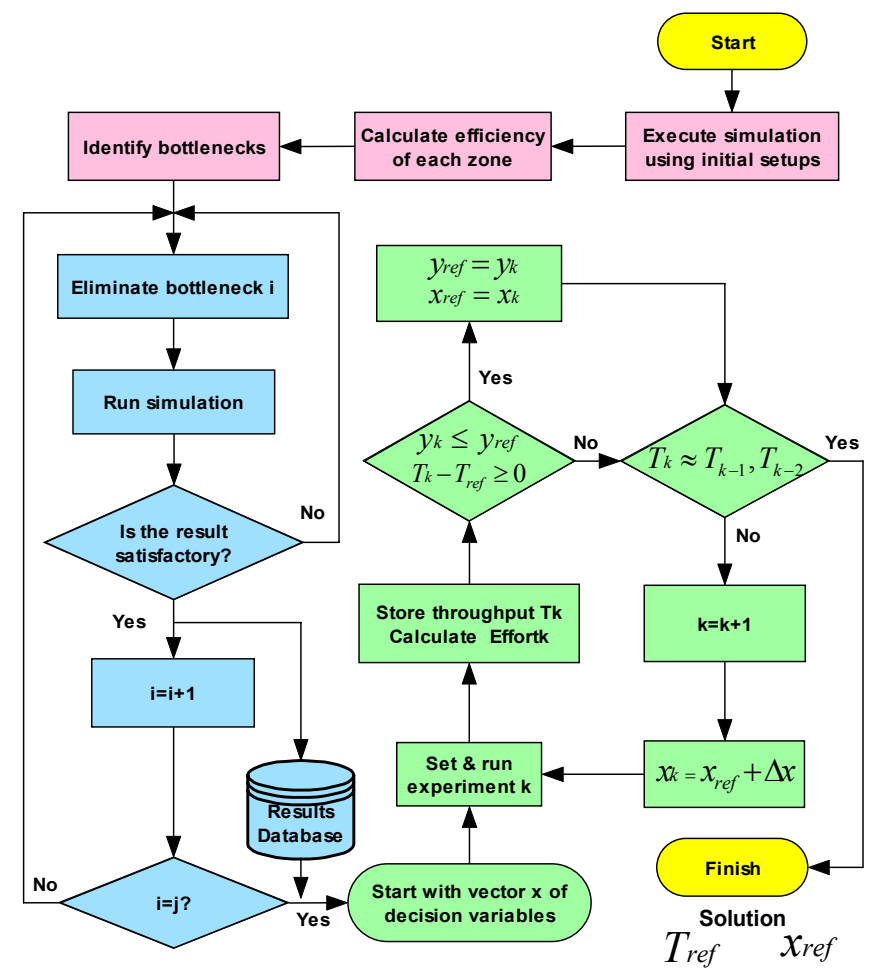

Figure 5: The proposed methodology

\subsection{Bottleneck Detection}

In order to facilitate the analysis, the assembly line was divided into a number of zones where each zone consisted of a several operations/workstations.

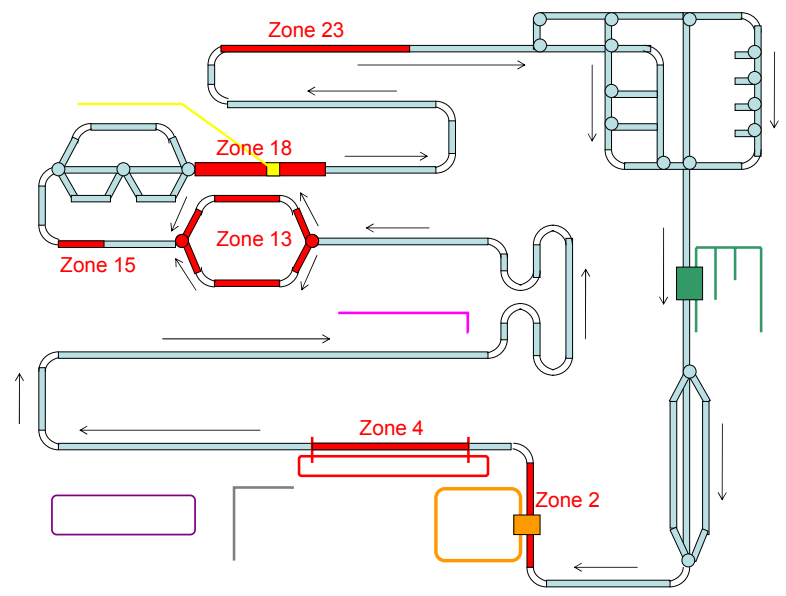

Figure 6: Bottlenecks locations

The bottleneck detection was carried out by analyzing each zone independently. When analyzing one zone, the three disturbances that affect the efficiency (machine breakdown, operator's performance and quality data) were deactivated at the rest of the zones so that the throughput of the line was only affected by this zone. The zones with the lowest efficiency levels (typically below $80 \%$ ) were considered the bottlenecks.

Having completed the analysis on each zone, it was found that the zone with the highest percentage of losses was zone 2 , followed by zone 4 . The possible cause for this was that most of manual operations did not achieve the standard performance. Further checks within Witness revealed many of the workstations in those zones were heavily blocked causing the idleness on the subsequent zones.

\subsection{Bottleneck Reduction/Elimination}

Having identified the bottleneck, changes were made on each zone by typically adding buffers and, whenever appropriate, modifying the cycle times of the workstations. The first sets of experiment aimed to improve the efficiency of the bottleneck zones individually to see the effect on the whole line. The ranking and selection method (Swisher et al. 2003) was used in this part and the best improvement was used to start the next phase. These experiments had been useful to identify the most sensitive zones and the operations/workstations that significantly affect the bottleneck.

Table 1: Bottleneck reduction/elimination summary

\begin{tabular}{|l|l|l|l|l|}
\hline Exp & Zone & Problems & Solutions implemented & $\begin{array}{l}\text { Eff.in- } \\
\text { creased }\end{array}$ \\
\hline 1 & 13 & $\begin{array}{l}\text { Diversion always } \\
\text { blocked. Starva- } \\
\text { tion at the begin- } \\
\text { ning and blocked } \\
\text { at the end. }\end{array}$ & $\begin{array}{l}\text { Cycle time Zones 12 } \\
\text { and 14 reduced. Buffer } \\
\text { added on the two } \\
\text { branches }\end{array}$ & $2.96 \%$ \\
\hline 2 & 15 & Human behavior & $\begin{array}{l}\text { Cycle time Zones 14 } \\
\text { and 15 reduced. Buffers } \\
\text { added before operations } \\
\text { with highest breakdown } \\
\text { rate }\end{array}$ & $6.29 \%$ \\
\hline 3 & 23 & Human behavior & $\begin{array}{l}\text { Buffer at the end of } \\
\text { CML. Cycle time re- } \\
\text { duced. }\end{array}$ & $1.09 \%$. \\
\hline 4 & 23 & Human behavior & $\begin{array}{l}\text { Human behavior deac- } \\
\text { tivated }\end{array}$ & $0.10 \%$ \\
\hline 5 & 18 & $\begin{array}{l}\text { CML always } \\
\text { blocked or sta- } \\
\text { tions broken down }\end{array}$ & $\begin{array}{l}\text { Buffer at the end of } \\
\text { CML. Cycle time re- } \\
\text { duced. }\end{array}$ & $0.72 \%$ \\
\hline 6 & 4 & Starvation & $\begin{array}{l}\text { Cycle time of manual } \\
\text { ops decreased. }\end{array}$ & $-0.51 \%$ \\
\hline 7 & 23 & Human behavior & $\begin{array}{l}\text { Buffers added in most } \\
\text { blocked work stations }\end{array}$ & $0.85 \%$ \\
\hline 8 & 18 & $\begin{array}{l}\text { CML always } \\
\text { blocked or sta- } \\
\text { tions broken down }\end{array}$ & $\begin{array}{l}\text { Buffers added in most } \\
\text { blocked work stations }\end{array}$ & $1.05 \%$ \\
\hline
\end{tabular}

Table 1 shows the first 8 experiments to investigate eight zones. The problems on each zone and the subsequent solution to improve the performance of that zone have also been listed. It can be seen that the most signifi- 


\section{Tjahjono and Fernández}

cant improvement is achieved by Experiment 2 which corresponds to zone 15 of the line.

In many cases, buffers can help reduce blocking, but buffers are costly and should be avoided whenever possible. For that reason, instead of adding buffers, updated breakdown data was used in the model. This means the reliability of equipment and operators in that zone was increased up to a certain level that the engineers considered as feasible for each workstation. The reliability data in this case include machine breakdown and human behavior (over cycles). This was believed to be more cost effective although required more focus on the maintenance of the workstations. A better maintenance would, in theory, reduce the downtime.

\subsection{Efficiency Improvement}

During this phase, the experiments were conducted in succession where the result from one experiment would be compared against the best result from the previous experiment (also known as the 'reference model'). This method has been made possible by the model reusability/composability principle within the simulation tool used in this study, where simulation models can be rapidly regenerated and modifications can be made as quickly as building the new ones.

If the results from an experiment is better than the result from the reference model, the reference model will be discarded and the results from new experiment will become the new reference model. On the contrary, if the result of the new experiment is worse than that of the reference model, then the results from new experiment will be discarded and the reference model is retained. The procedure will repeat until the throughput does not increase significantly and/or the effort needed to improve the throughput is no longer worthwhile.

For this reason, an objective comparison between one set of parameters to another would need to consider effort or investment needed in order to determine whether or not a result is satisfactory, that is to estimate how much the potential investment would cost. If this investment is too high compared to the improved throughput, the model is discarded. On the other hand, if the throughput achieved compensates the investment needed, the model is taken as a reference for the next improvement.

The sets of variable used in the experimentation are:

$T_{k}$ : throughput of the experiment $k$

$T_{\text {ref }}$ : throughput of the model taken as reference

$T_{0}$ : throughput of the initial model

$x_{k}$ : configuration of the model of the experiment $k$

$x_{r e f}$ : configuration of the model taken as reference

Weighting of variables is used to decide whether or not the model is worthy in terms of effort or investment. These input variables can be grouped into: cycle time, ma- chine reliability and buffer allocation (or removal). These variables will be included into the calculation of effort and each of them has weighting factor. The weighting factors were discussed with manufacturing engineers and agreed by the management.

Reducing the cycle time of a zone implies the addition of extra workforce so that it involves a substantial investment, hence the largest weighting. Furthermore, according to the engineers, in order to see to effect of reducing the cycle time, the plant needs to be shut down for at least one week to carry out the changes.

As mentioned before, adding buffers is not always feasible and requires a significant investment. In the case of an assembly line connected by conveyor belts, a buffer could mean additional length of the conveyor so that it can accommodate more engines. For this reason, the weighting was considered medium.

Improving machine reliability through a better maintenance was considered the most cost effective, hence the most feasible option. This is because the effort only requires a better maintenance, which is reasonably easy to achieve.

By combining the three factors above, the following equation can be used to determine the total effort needed for an improvement obtained from an experiment:

$$
\text { Effort }_{k}=W_{C T} \times C T_{k}+W_{B D} \times B D_{k}+W_{B F} \times B F_{k}
$$

where

$C T_{k}=$ number of zones whose cycle time reduced

$B D_{k}=$ number of work stations whose reliability data improved

$B F_{k}=$ number of buffer added (or removed)

$W_{C T}=50 \%$

$W_{B D}=15 \%$

$W_{B F}=35 \%$

The trade-off between the efficiency improvement achieved and the effort needed to implement that improvement is denoted using the improvement index $(y)$ :

$$
y_{k}=\frac{\text { Effort }_{k}}{T_{k}-T_{0}}
$$

where

$y_{k}=$ improvement index at experiment $k$

Effort $_{k}=$ effort required to realize $T_{k}$

At each experiment, $y_{k}$ is compared against $y_{\text {ref }}$.

If

$\quad y_{k} \leq y_{r e f}$ and $T_{k}-T_{r e f} \geq 0$
then $y_{r e f}=y_{k}$ and $x_{r e f}=x_{k}$

Using this rule, the effort associated to the model together with its throughput is taken into account. When the whole experiments are completed, the most feasible solution should be $y_{r e f}$ with the configuration of $x_{r e f}$. 
Table 2 lists the data from the experimentation and shows all the calculations extracted from a spreadsheet to come to the final result. Experiments 9, 12, 13, 16, 17, 18 indicate the models whose experiments were considered as reference for the next experiments and Experiment 21 is the final solution. As it is planned to reduce the cycle time of the actual line, it was decided not to discard experiments 16,17 and 18 in order to meet this requirement.

Table 2: Results from efficiency improvement stage

\begin{tabular}{|c|c|c|c|c|c|c|c|c|c|}
\hline \multirow{2}{*}{$\begin{array}{l}\text { Exp } \\
\text { (k) }\end{array}$} & \multirow{2}{*}{$T_{k}-T_{0}$} & \multirow{2}{*}{$T_{k}-T_{r e f}$} & \multicolumn{3}{|c|}{ Modification } & \multirow{2}{*}{ Effort $_{k}$} & \multirow{2}{*}{$y_{k}$} & \multirow{2}{*}{$\begin{array}{l}\text { Ref } \\
\text { model }\end{array}$} & \multirow{2}{*}{$\begin{array}{l}\text { Efficiency } \\
\text { Increase }\end{array}$} \\
\hline & & & $\mathrm{BD}$ & CT & $\mathrm{BF}$ & & & & \\
\hline 0 & 0.00 & 0 & & & & 0 & 0.00 & 0 & $0.00 \%$ \\
\hline 9 & 2.51 & 2.51 & 4 & & & 120 & 47.75 & 0 & $5.60 \%$ \\
\hline 10 & 0.01 & -2.50 & & & 10 & 820 & 61500 & 9 & $0.03 \%$ \\
\hline 11 & 0.25 & -2.27 & 25 & & & 870 & 3527.03 & 9 & $0.55 \%$ \\
\hline 12 & 6.09 & 3.58 & 4 & & & 240 & 39.42 & 9 & $13.57 \%$ \\
\hline 13 & 6.58 & 4.07 & & & -1 & 170 & 25.84 & 12 & $14.66 \%$ \\
\hline 14 & 6.58 & 0.00 & 14 & & & 590 & 89.67 & 13 & $14.66 \%$ \\
\hline 15 & 6.54 & -0.04 & 1 & & & 200 & 30.59 & 13 & $14.57 \%$ \\
\hline 16 & 9.13 & 2.55 & 23 & 4 & 2 & 1400 & 153.34 & 13 & $20.35 \%$ \\
\hline 17 & 11.36 & 2.23 & & 7 & & 2100 & 184.80 & 16 & $25.33 \%$ \\
\hline 18 & 14.52 & 3.16 & & 13 & & 3400 & 234.13 & 17 & $32.36 \%$ \\
\hline 19 & 15.08 & 0.56 & & 1 & 12 & 4340 & 287.80 & 18 & $33.61 \%$ \\
\hline 20 & 14.17 & -0.35 & & & & 3705 & 261.44 & 18 & $31.58 \%$ \\
\hline 21 & 15.46 & 0.94 & 3 & & & 3490 & 225.70 & 18 & $34.46 \%$ \\
\hline 22 & 16.06 & 0.60 & & & 6 & 3910 & 243.41 & 21 & $35.80 \%$ \\
\hline 23 & 14.41 & 1.05 & & & 3.5 & 3735 & 259.14 & 21 & $32.12 \%$ \\
\hline 24 & 14.56 & -0.90 & & & 2 & 3845 & 264.02 & 21 & $32.46 \%$ \\
\hline
\end{tabular}

Figure 7 plots the results from the experiments in terms of efficiency improvement, starting from the initial model (Experiment 0). From this chart, the largest increase in efficiency is given by Experiment 22.

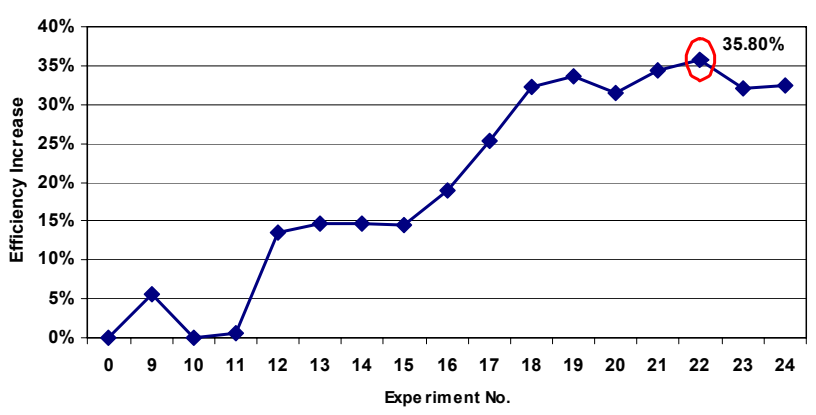

Figure 7: Efficiency increase

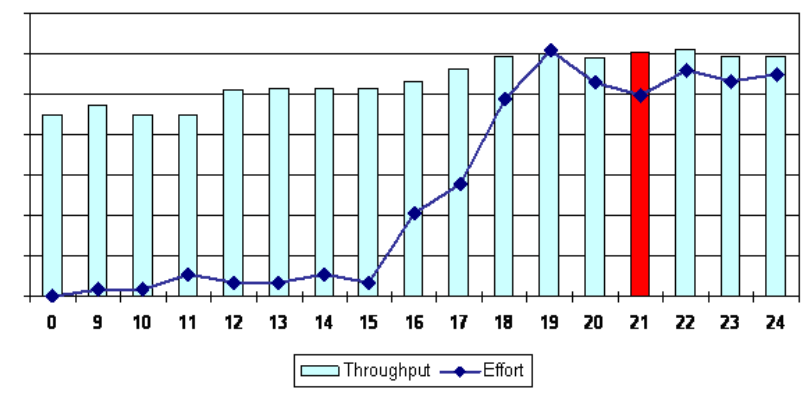

Figure 8: Throughput vs. effort needed

To test this solution, the increased efficiency was weighed up against the potential investment/effort required to achieve that solution. The effort was calculated using Equation (4), and Equation (5) compared the two experiments. Figure 8 takes into account these 2 factors. Although the result from Experiment 22 was the best, due to the significant investment needed, the result from Experiment 21 in fact gave more feasible solution.

\section{CONCLUSION}

A methodology has been proposed to assist experimentation procedures. The methodology entails the 3 key stages to increase productivity of the line namely: bottleneck detection, bottleneck reduction/elimination and efficiency improvement. The first two, to some extent, share similar characteristics with many bottleneck detection and reduction methods exist in the literature (e.g. Law and Kelton 1991; Roser et al. 2001; Roser et al. 2002; Ingemansson et al. 2005; Faget et al. 2005), and from practical point of view, this was deemed sufficient. The last stage of the methodology is particularly useful in order to decide the most feasible solution from a series of experiment results. Using the methodology, it can be concluded that the best solution did not necessarily mean the highest increase in efficiency. In practice, key constraints such as effort/investments need to be taken into account, so as to maximize the throughput and minimize the effort to implement that solution.

From the theoretical perspective, to some extent, the methodology proposed in this paper has provided an alternative method to experimentation design. Not only can the methodology be used within a simulation study of an assembly line, it can also be potentially used in other simulation models where queues/bottlenecks affect the throughput and where there are constraints that make it difficult to implement the solution.

The limitation of this approach is perhaps due to the fact that the model is built using a secondary user interface based on Excel, and all the necessary information, such as breakdown data are already available. This means the methodology works well if the simulation model is highly reusable, so discarding the infeasible model after an experiment and generating the new one for the next experiment are both quick and straightforward. Another reason is that the outputs of the model are also available in a ready to use format, so comparison of the throughputs can be done easily.

Investigation will be needed to further develop the criteria for the weightings, which at the time of the study, was based purely on the judgment of the manufacturing engineers and managers. Nonetheless, the study has provided a deeper understanding of the possible causes for poor performance of the assembly line, and the findings have helped the manufacturing engineers at the company identify the most feasible sets of parameters in order to improve efficiency and productivity. 


\section{REFERENCES}

Baines, T.S., R.E. Asch, L. Hadfield, J.P. Mason, S.R. Fletcher, and J.M. Kay. 2005. Towards a theoretical framework for human performance modelling within manufacturing systems design. Simulation Modelling Practice And Theory 13:486-504.

Chryssolouris, G. 2005. Manufacturing systems: theory and practice. 2nd ed. New York: Springer.

Faget, P., U. Eriksson, and F. Herrmann. 2005. Applying Discrete Event Simulation and an Automated Bottleneck Analysis as an Aid to Detect Running Production Constraints, In Proceedings of the 2005 Winter Simulation Conference, 1401-1407.

Guru, A., and P. Savory. 2004. A Template-based Conceptual Modeling Infrastructure for Simulation of Physical Security Systems. In Proceeding of the 1994 Winter Simulation Conference, 866-873.

Ingemansson, A., and G.S. Bolmsjo. 2004. Improved efficiency with production disturbance reduction in manufacturing systems based on discrete-event simulation. Journal of Manufacturing Technology Management 15 (3):267-79.

Ingemansson, A., T. Ylipaa, and G.S. Bolmsjo. 2005. Reducing bottlenecks in a manufacturing system with automatic data collection and discrete-event simulation. Journal of Manufacturing Technology Management 16(6):615-28.

Kuivanen, R. 1996. Disturbance control in flexible manufacturing. International Journal of Human Factors in Manufacturing 6(1):41-56.

Law, A., and W.D. Kelton. 1991. Simulation Modeling and Analysis. 2nd edition. New York: McGraw-Hill.

Nakayama, M. K. 2006. Output analysis for simulations. In Proceedings of the 2006 Winter Simulation Conference, 36.

Reid, R., and N. Sanders. 2005. Operations Management: An Integrated Approach. John Wiley \& Sons.

Roser, C., M. Nakano, and M. Tanaka. 2001. A practical bottleneck detection method. In Proceedings of the 2001 Winter Simulation Conference, 949-953.
Roser, C., M. Nakano, and M. Tanaka. 2002. Shifting bottleneck Detection. In Proceedings of the 2002 Winter Simulation Conference, 1079-1086.

Sargent, R. G. 2004. Validation and verification of simulation models. In Proceedings of the 2004 Winter Simulation Conference, 17.

Swisher, J. R., S.H. Jacobson, and E. Yücesan. 2003. Discrete-event simulation optimization using ranking, selection, and multiple comparison procedures: A survey. ACM Trans. Model. Comput. Simul., 134-154.

Winnell, A., and J. Ladbrook. 2004. Towards composable simulation: Supporting the design of engine assembly lines. In Proceedings of the OR Simulation Study Group 2-day Workshop, 311-318.

\section{AUTHOR BIOGRAPHIES}

BENNY TJAHJONO is the Director of Manufacturing Masters Program at Cranfield University. He holds a first degree in Electrical Engineering, an MSc and a $\mathrm{PhD}$ both in Manufacturing Systems Engineering from Cranfield University. His research interest is in simulation modeling and analysis of manufacturing systems. $\mathrm{He}$ is currently leading a research team to work on the EPSRC-funded project entitled An Evolutionary Approach to Rapid Development of Simulation Models (grant no EP/E037631/1). Dr Tjahjono has been working closely with global companies, such as Rolls-Royce, GlaxoSmithKline, Ford and Airbus, on several funded projects associated with the decision making process using discrete-event simulation.

RAÚL FERNÁNDEZ received an MSc in Knowledge Management for Innovation from Cranfield University, and a Masters degree in Industrial Engineering from Polytechnic University of Madrid, Spain. In addition to working on this project, he was part of the team working on a project sponsored by Airbus UK to capture and structure the knowledge in designing and costing aircraft wings and fuselage. 